\title{
Serum Neutrophil Gelatinase-associated Lipocalin in children with septicemia
}

\author{
Mohamed A. Kassem*, MostafaAshry*, Ahmed Noor Eldein** \\ Department of Pediatrics, Faculty of Medicine, Sohag University, Sohag, Egypt* \\ ** Department of Clinical Pathology, Faculty of Medicine, Sohag University, Sohag, \\ Egypt
}

\begin{abstract}
Neutrophil gelatinase-associated lipocalin (NGAL) is a rapidly emerging biomarker for early detection of acute kidney injury (AKI). The aim of the study is to evaluate the impact of sepsis on serum NGAL in critically ill children, and to investigate whether the presence of sepsis affects the ability of serum NGAL to predict AKI. Sixty eight patients, who met criteria of sepsis and related syndromes, were classified into two groups (septic shock and severe sepsis). They were reclassified regarding who developed AKI into AKI and non- AKI groups. Twenty sex and age matched healthy subjects served as a control group. Serum NGAL was assayed using Enzymelinked Immunosorbent Assay (ELISA), and serum creatinine was measured using kinetic spectrophotometric method. Serum NGAL levels were significantly high in critically ill septic patients compared to healthy controls (median: $100.4 \mathrm{ng} / \mathrm{ml}$ vs. 47.1 $\mathrm{ng} / \mathrm{ml}, \mathrm{P}<0.0001$ ), and were significantly higher in septic shock (median $105.1 \mathrm{ng} / \mathrm{ml}$ ) than in severe sepsis (median $96 \mathrm{ng} / \mathrm{ml}$ ) with $\mathrm{P}$ values of 0.005 . However, there was no significant difference in the levels of serum NGAL between AKI patients and nonAKI patients $(\mathrm{P}=0.3)$. Receiver operating characteristic (ROC) curve analysis of serum NGAL for prediction of AKI in critically ill septic children showed an area under the curve (AUC) was 0.56 (95\% C.I. $=0.42-0.70)$ with an optimal cutoff value of $102.5 \mathrm{ng} / \mathrm{ml}$, sensitivity $=56.2 \%$, specificity $55.6 \%$, PPV $=52.9 \%, \mathrm{NPV}=58.8 \%$, and accuracy $=55.9 \%$. For serum creatinine, the AUC was $0.97(95 \%$ C.I. $=0.94-1.00)$ with an optimal cutoff value of $0.6 \mathrm{mg} / \mathrm{dl}$, sensitivity $=90.6 \%$, specificity $91.7 \%$, $\mathrm{PPV}=90.6 \%, \mathrm{NPV}=91.7 \%$ and accuracy $=91.2 \%$. In conclusion, serum NGAL is raised in critically ill septic children and is a marker of bacterial infection and systemic inflammation. However, in AKI associated with sepsis, serum NGAL is not a specific biomarker for the prediction of AKI and it loses its early predictive property. In such patients, serum creatinine is more specific than serum NGAL.

Key words: Neutrophil gelatinase-associated lipocalin, Acute kidney injury,Creatinine, Sepsis.
\end{abstract}

\section{INTRODUCTION}

Acute kidney injury is common in critically ill children admitted to intensive care unit. The etiology of acute kidney injury is multifactorial and the incidence varies between 1 and $41 \%$ (Alkandari et al., 2011) and (Krishnamurthy, 2013) probably due to the different definitions used in clinical studies.Sepsis remains a significant risk factor and it is the leading cause acute kidney injury in critically ill children

(Shalaby et al., 2014). There is an association of acute kidney injury with sepsis in $66.7 \%$ and $48.2 \%$ progress to septic shock (Consuelo et al., 2012).

Unfortunately, Serum Creatinine (the main acute kidney injury biomarker used in the clinical setting) is a late marker of reduced glomerular filtration rate, which limits ability to detect acute kidney injury early and to initiate 
SOHAG MEDICAL JOURNAL Serum Neutrophil Gelatinase-associated Lipocalin Vol. 23 No.2Apr 2019

Mohamed A. Kassem.et al

clinical therapeutic studies. Therefore, Diagnostic specificity and sensitivity of new biomarkers are currently weighed against creatinine-based criteria (Waikar et al., 2009).

Several proteins and biochemical markers emerged as sensitive and specific biomarkers capable of the early detection of acute tubular injury (Devarajan , 2011). The most famous of these biomarkers is neutrophil gelatinase-associated lipocalin (NGAL).

Neutrophil gelatinase-associated lipocalin (NGAL) is a rapidly emerging biomarker for early detection of AKI. Seemingly, NGAL levels in both plasma and urine can be used to detect AKI days before creatinine at least when the time of insult to the kidneys is known, Wagener et al., (2006).

Before introducing NGAL as an early AKI marker in pediatric ICU, it is vital to clarify the effects of infection/ sepsis on serum NGAL in patients with and without AKI. So, the aim of this work is to study the impact of sepsis on the level of serum NGAL in critically ill pediatric patients and to evaluate whether the presence of sepsis affects the ability of serum NGAL to predict AKI.

\section{MATERIALS AND METHODS}

This is a prospective observational study that was conducted in Pediatric Intensive Care Unit (PICU) and emergency room in Sohag University hospital, from January 2018 to December 2018.

Sixty eight children with septicemia were included in this study. Sepsis was diagnosed according to the definitions of the American College of Chest Physicians/Society of Critical Care Medicine modified specifically for pediatrics. , Goldstein et al., (2005). The criteria used for diagnosis of septicemia in this study were:

1. Core temperature of $>38.5^{\circ} \mathrm{C}$ or $<36^{\circ} \mathrm{C}$. (axillary method was used in measuring the temperature)

2. Tachycardia.

3. Tachypnea.

4. Leukocyte count elevated or depressed for age or $>10 \%$ immature neutrophils.

5. Positive blood culture.

This is in addition to the manifestations of the primary disease.

Septic shock was diagnosed when clinical signs of inadequate tissue perfusion including any of the following:

1-Decreased or altered mental status.

2-Prolonged capillary refill of more than 2 seconds.

3-Diminished pulses, mottled cool extremities.

4-Decreased urine output of less than 1 $\mathrm{ml} / \mathrm{kg}$ per hour.

5-Persistent hypotension despite of adequate fluid resuscitation.

This is in addition to the manifestations of septicemia.

Septic patients were reclassified into two groups regarding the diagnosis of acute kidney injury. AKI was defined using the creatinine according to the Kidney Disease Improving Global Outcomes (KDIGO) guidelines for acute kidney injury published in 2012 . Twenty healthy subjects, age and sex matched, served as a control group.

Inclusion criteria:

All Children < 12 years of age admitted to the emergency room and Pediatric intensive care unit, and meeting criteria for septicemia were included in the study.

Exclusion criteria:

1. Neonates (age less than 28 days)

2. Children with a history of kidney disease. 
SOHAG MEDICAL JOURNAL Serum Neutrophil Gelatinase-associated Lipocalin Vol. 23 No.2Apr 2019

Mohamed A. Kassem.et al

3. Children with evidence of kidney disease

Clinical data were collected (demographic, diagnosis on admission,inotropic drugs, length of stay and outcome).For each patient, the severity of illness was calculated using the PRISM III (Pediatric risk of mortality) score of Pollack et al., (1996).

Informed consents to participate in the study were obtained from all subjects' parents. The study was approved by Sohag University Ethical Committees.

\section{Laboratory Methodology:}

Samples Collection:

Venous blood samples were withdrawn from all subjects on plain bloodcollection tubes. The obtained samples were centrifuged at $3000 \mathrm{xg}$ for 15 minutes, and serum was stored at $20^{\circ} \mathrm{C}$ until analysis. Samples were thawed and mixed thoroughly just prior to the assay to avoid erroneous results of repeated freeze/thaw cycles, hemolyzed and lipemic samples were avoided. Serum Neutrophil gelatinaseassociated lipocalin (NGAL) was assayed by Enzyme- linked Immunosorbent Assay (ELISA) and serum creatinine was assayed using the enzymatic spectrophotometric method.

\section{Assay of Serum Creatinine:}

Serum creatinine levels were measured by using the kinetic spectrophotometric method according to Jaffe' method without deproteinization, Mazzachi et al., (2000). This is a compensated method based on manufacturer's instructions (Roche Diagnostics GmbH, Mannheim, Germany).

Assay of Serum NGAL:

Serum NGAL was assayed according to manufacturer's protocol using Human Lipocalin-2/NGAL ELISA kit (BioVendor Laboratory Medicine. Inc., Cat. No. RD191102200R) for the quantitative measurement of human Lipocalin-2/NGAL. The standards, quality controls and samples were incubated in micro titer wells precoated with polyclonal anti-human Lipocalin-2 antibody. Then biotinlabeled polyclonal anti-human Lipocalin-2 antibody was added and incubated with captured Lipocalin-2. After washing away any unbound biotinylated antibody, the streptavidinHRP conjugate was added. Following a wash to remove any unbound antibody enzyme reagent, the remaining conjugate was allowed to react with the substrate solution (TMB) resulting in yellow color development proportional to the amount of NGAL bound in the initial step. The color development was stopped by addition of acidic solution and the intensity of the color was proportional to the concentration of Lipocalin-2. The absorbance of the resulting yellow color was measured spectrophotometrically at $450 \mathrm{~nm}$. A standard curve was constructed by plotting absorbance values against concentrations of the standards, and concentrations of the samples were determined using this curve, Mori et al., (2007).

Statistical Analysis:

Data was analyzed using Statistical Package for Special Science software computer program version 16.0 (SPSS Inc., Chicago, Illinois, USA). Categorical variables were expressed as number (n), percent $(\%)$ and were compared using the Chi square test or Fischer's Exact tests, as indicated. Continuous variables were compared using Mann-Whitney test and KruskalWallis ANOVA, as indicated. The associations between variables were assessed by Spearman rank order correlation analysis. In order to measure the sensitivity, specificity, 
SOHAG MEDICAL JOURNAL Serum Neutrophil Gelatinase-associated Lipocalin Vol. 23 No.2Apr 2019

Mohamed A. Kassem.et al

positive predictive value (PPV), and biomarker, Hanley and McNeil (1982). negative predictive value (NPV) of serum NGAL for the prediction of AKI, receiver operating characteristic (ROC) curve was generated and the area under the curve (AUC) was calculated. An AUC of 0.5 is no better than expected by chance, whereas a value of 1.0 signifies a perfect Multivariate regression analysis was used to test the association between multiple quantitative and qualitative independent variables with the dependent variable. $\mathrm{P}$ value less than or equal 0.05 was considered statistically significant.

\section{Results}

Patients were grouped into severe sepsis and septic shock according to the definitions of the American College of Chest Physicians/Society of Critical Care Medicine modified specifically for pediatrics. Different septic patients' groups are shown in table (1). This table shows that patients with septic shock are much younger than patients with sepsis with no statistically significant difference as regard sex between the two groups. Patients with septic shock have higher serum creatinine $(p<0.0001)$ and lower estimated GFR $(\mathrm{P}<0.0001)$ than septic patients without shock with statistically significant difference. NGAL is higher in patients with septic shock $(105.14 \pm 6.11 \mathrm{ng} / \mathrm{ml})$ when compared to septic patients without shock $(96.66 \pm 11.53 \mathrm{ng} / \mathrm{ml})$ with statistically significant difference $(\mathrm{p}=0.0005)$. This table also shows statistically significant difference between septic patients with septic shock and those without shock as regard mortality [66\% vs. 8\%], PRISM score [21 vs.9.5], length of hospital stay [9 vs. 12days].

Serum NGAL level was significantly increased in critically ill children with septic shock [mean: $105.14 \pm 6.11 \mathrm{ng} / \mathrm{ml}$ ] compared to either critically ill children with severe sepsis [mean96.66 $\pm 11.53 \mathrm{ng} / \mathrm{ml}), \quad \mathrm{P}=0.005]$ or to healthy controls [(mean47.16 $\pm 5.64 \mathrm{ng} / \mathrm{ml}) \mathrm{p}<0.0001]$ (Figure 1 \&table1).

Multivariate regression analysis showed that septic shock was the only independent variable for elevation of serum NGAL, $\mathrm{P}=0.006$ (Table 2).

AKI was developed in $32(47.06 \%)$ out of 68 critically ill patients, there was no significant difference in the level of serum NGAL between patients with AKI compared to those without AKI $(\mathrm{P}=0.3)$. In consistence with the definition of AKI used in this study, serum creatinine was significantly increased in critically ill children with AKI $(\mathrm{P}<0.0001)$ (Table 3). 
SOHAG MEDICAL JOURNAL Serum Neutrophil Gelatinase-associated Lipocalin Vol. 23 No.2Apr 2019 Mohamed A. Kassem.et al

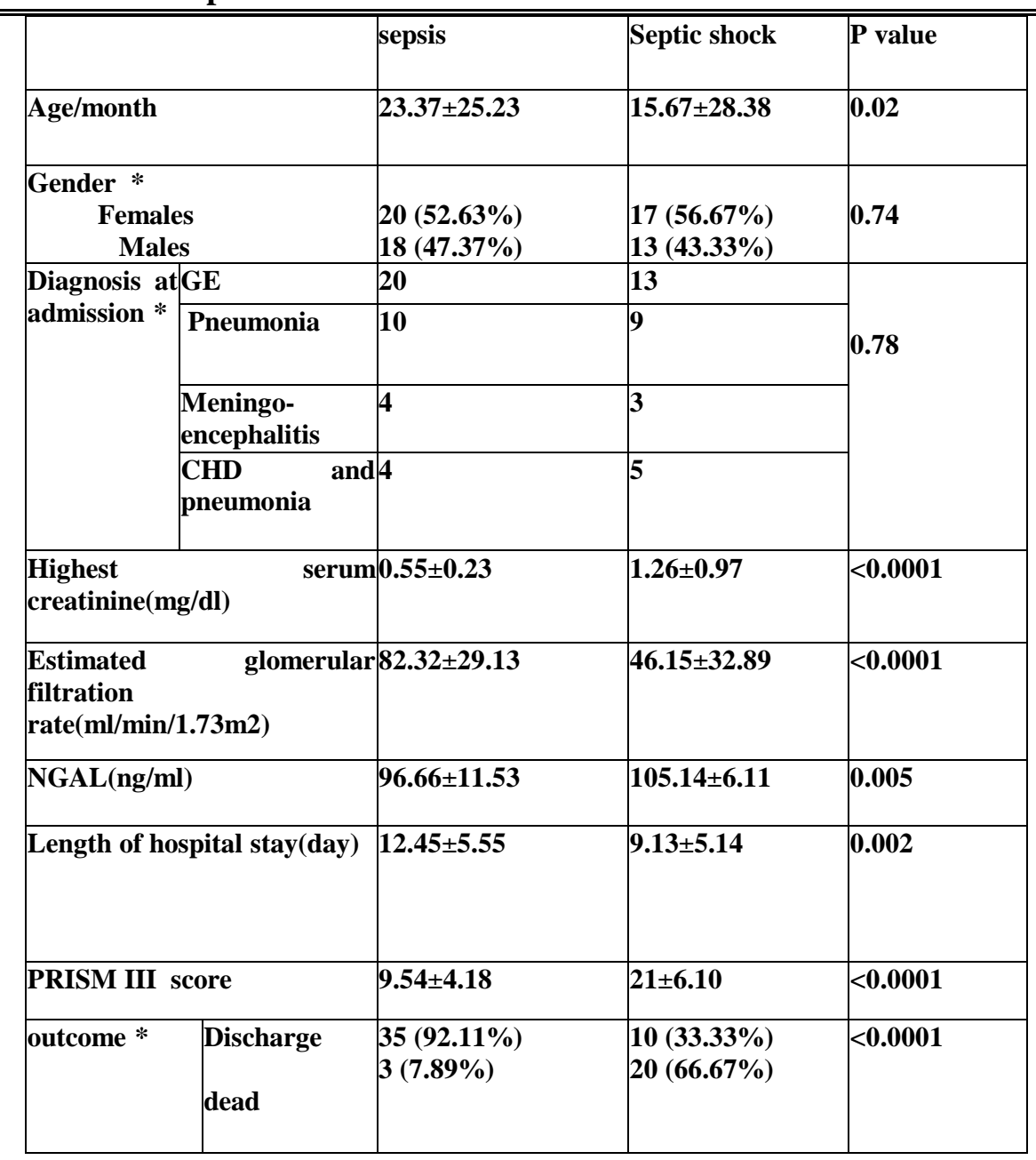

Table 1: Comparison between different septic patients' groups

*Data are represented as mean and standard deviation except* represented as frequency. PRISM III=

pediatric risk of mortality, NGAL= neutrophil gelatinase- associated lipocalin $\mathrm{CHD}=$ congenital heart diseas

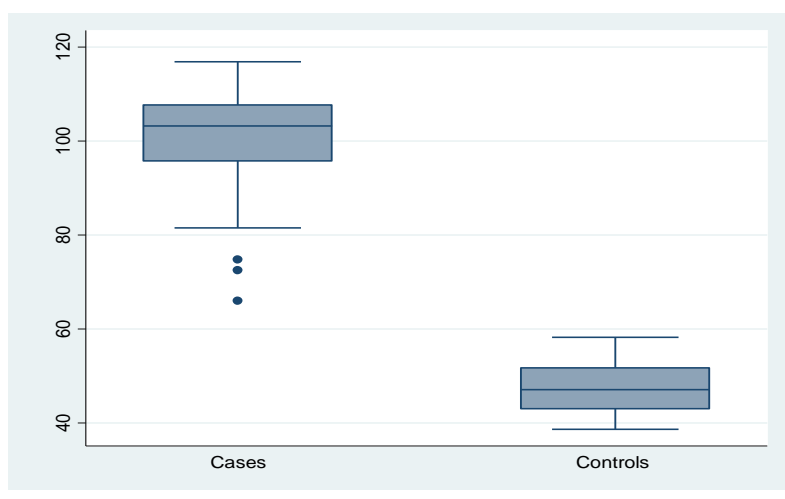

Figure (1) comparison between cases and controls according to serum NGAL 
SOHAG MEDICAL JOURNAL Serum Neutrophil Gelatinase-associated Lipocalin Vol. 23 No.2Apr 2019

Mohamed A. Kassem.et al

\begin{tabular}{|c|c|c|c|c|}
\hline Variables & $\begin{array}{l}\text { Unadjusted regression } \\
\text { co-efficient } \\
(95 \% \mathrm{CI})\end{array}$ & $P$ value & $\begin{array}{l}\text { Adjusted regression } \\
\text { co-efficient } \\
(95 \% \text { CI })\end{array}$ & $P$ value \\
\hline Age/month & $-0.04(-0.13: 0.06)$ & $\mathbf{0 . 4 2}$ & $-0.07(-0.18: 0.04)$ & 0.22 \\
\hline gender & $0.86(-0.4 .22: 5.93)$ & 0.74 & $0.12(-4.94: 5.18)$ & 0.96 \\
\hline Highest serum creatinine & $2.81(-0.52: 6.14)$ & 0.10 & $2.64(-3.59: 8.89)$ & 0.40 \\
\hline $\begin{array}{ll}\text { Estimated } & \text { glomerular } \\
\text { filtration rate }\end{array}$ & $-0.05(-0.12: 0.03)$ & 0.21 & $0.06(-0.09: 0.22)$ & 0.40 \\
\hline Septic shock & $8.50(3.84-13.15)$ & 0.001 & $10.40(3.11: 17.70)$ & 0.006 \\
\hline Acute kidney injury & $2.63(-2.40: 7.66)$ & 0.30 & $1.22(-7.52: 9.96)$ & 0.78 \\
\hline Low urine output & $5.99(-1.00: 12.98)$ & 0.10 & $-4.65(-25.49: 16.19)$ & 0.66 \\
\hline hypervolemia & $6.85(-0.42: 14.12)$ & 0.07 & $15.13(-9.94: 40.20)$ & 0.23 \\
\hline Pulmonary edema & $11.37(-3.34: 26.09)$ & 0.13 & $7.68(-8.59: 23.95)$ & 0.35 \\
\hline Mechanical ventilation & $3.76(-1.41: 8.92)$ & 0.15 & $0.42(-15.73: 16.59)$ & 0.96 \\
\hline Death & $2.90(-2.39: 8.21)$ & 0.28 & $-5.00(-21.01: 11.06)$ & 0.54 \\
\hline
\end{tabular}

Table (2) Multivariate regression analysis shows that septic shock is the only independent variable for elevation of serum NGAL $(p=0.006)$

\begin{tabular}{|c|c|c|c|}
\hline & Non AKI & AKI & P VALUE \\
\hline Number & $36(52.94 \%)$ & $32(47.06 \%)$ & \\
\hline AGE(month) & $29.41 \pm 32.16$ & $9.34 \pm 12.57$ & 0.0001 \\
\hline Highest serum creatinine (mg/dl) & $0.46 \pm 0.13$ & $1.32 \pm 0.89$ & $<0.0001$ \\
\hline $\begin{array}{l}\text { Estimated glomerular filtrati } \\
\text { rate }(\mathrm{ml} / \mathrm{min} / 1.73 \mathrm{~m} 2)\end{array}$ & $93.63 \pm 23.28$ & $35.68 \pm 16.90$ & $<0.0001$ \\
\hline NGAL(ng/ml) & $99.17 \pm 11.23$ & $101.80 \pm 9.29$ & 0.30 \\
\hline $\begin{array}{l}\text { Urine output * } \\
\text { Normal } \\
\text { Low }\end{array}$ & $\begin{array}{l}36(100 \%) \\
0\end{array}$ & $\begin{array}{l}22(68.75 \%) \\
10(31.25 \%)\end{array}$ & $<0.0001$ \\
\hline \begin{tabular}{|l|} 
Signs of Hypervolemia* \\
No \\
Yes \\
\end{tabular} & $\begin{array}{l}36(100 \%) \\
0\end{array}$ & \begin{tabular}{|l}
$23(71.88 \%)$ \\
$9(28.13 \%)$
\end{tabular} & 0.001 \\
\hline PRISM III score & $9.64 \pm 4.40$ & $20.17 \pm 6.65$ & $<0.0001$ \\
\hline Length of stay (day) & $12.33 \pm 5.5$ & $9.47 \pm 5.35$ & 0.007 \\
\hline $\begin{array}{l}\text { Outcome* } \\
\text { Good } \\
\text { Dead }\end{array}$ & $\begin{array}{l}\text { 33(91.67\%) } \\
\text { 3(8.33\%) }\end{array}$ & $\begin{array}{l}12(37.50 \%) \\
20(62.50 \%)\end{array}$ & $<0.0001$ \\
\hline
\end{tabular}

Table 3: Clinical data of critically ill children (AKI patients vs. non- AKI patients).

*Data are represented as mean and standard deviation except* represented as frequency.

PRISM III=pediatric risk of mortality, NGAL= neutrophil gelatinase- associated lipocalin 


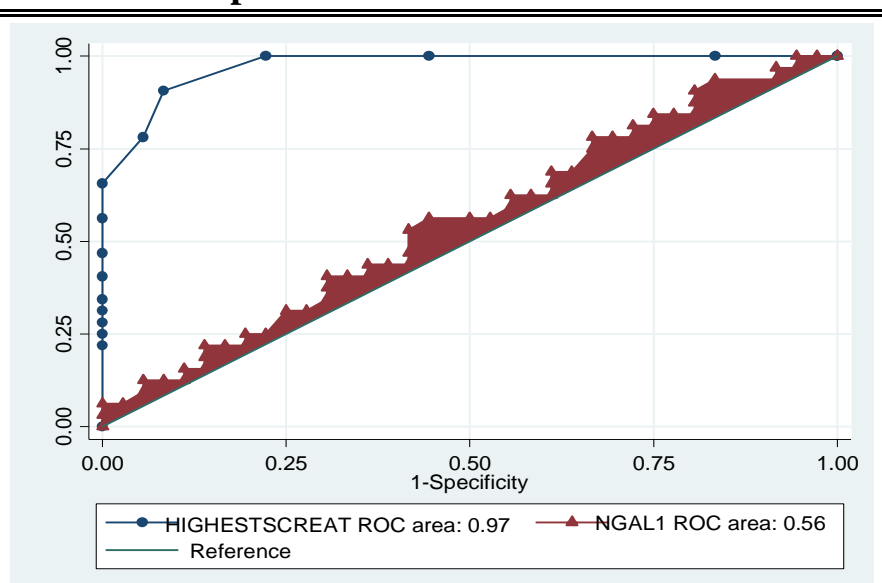

Figure (2) Comparison between Receiver operating characteristic (ROC) curve analysis of serum NGAL and serum creatinine concentrations for prediction of AKI in cases. $(\mathbf{p}<0.0001)$

Figure 2 shows the receiver operating characteristic (ROC) curve analysis of serum NGAL and serum creatinine for prediction of AKI in critically ill septic children. For serum NGAL, the area under the curve (AUC) was 0.56 (95\% C.I. $=0.42-0.70)$ with an optimal cutoff value of $102.5 \mathrm{ng} / \mathrm{ml}$, sensitivity $=56.2 \%$, specificity $55.6 \%$, PPV $=52.9 \%, \mathrm{NPV}=58.8 \%$, and accuracy $=55.9 \%$. For serum creatinine, the AUC was 0.97 (95\% C.I. $=0.94-1.00)$ with an optimal cutoff value of $0.6 \mathrm{mg} / \mathrm{dl}$, sensitivity $=90.6 \%$, specificity $91.7 \%, \mathrm{PPV}=90.6 \%, \mathrm{NPV}=91.7 \%$ and accuracy $=91.2 \%$.

\section{Discussion}

AKI is common among critically ill children and early diagnosis is vital to prevent and decrease associated morbidity and mortality. Sepsis remains a significant risk factor and it is the leading cause of acute kidney injury in critically ill children (Shalaby et al., 2014).

Neutrophil gelatinase-associated lipocalin (NGAL) is a rapidly emerging biomarker for early detection ofAKI. NGAL is a $25 \mathrm{KDa}$ protein that is expressed at very low concentrations in several human tissues including the kidney, lungs, and gastrointestinal tract, Kjeldsen et al., (2000). It is released by neutrophils upon activation and it is a marker of bacterial infection and systemic inflammation, Mori et al., (2005).

In this study, we evaluated serum NGAL as a biomarker of sepsis and as a specific predictor for AKI in septic children. We found that, serum NGAL was significantly increased in critically ill children with different grades of sepsis (severe sepsis and septic shock) compared to healthy controls. Also, serum NGAL in critically ill children with septic shock was significantly higher than in critically ill children with severe sepsis, reflecting the severity of illness in those patients.

Our results in accordance with Singer E. et al, 2013 who declared that NGAL has important limitations in early detection of AKI, including its responsiveness in systemic inflammation, which is partially uncoupled from its response to kidney injury and which needs to be considered when interpreting NGAL results clinically. The picture in critically ill septic patients was somewhat less clear, since sepsis is an important inducer of blood and urinary 
SOHAG MEDICAL JOURNAL Serum Neutrophil Gelatinase-associated Lipocalin Vol. 23 No.2Apr 2019

Mohamed A. Kassem.et al

NGAL expression even in the absence of changes in creatinine. In sepsis, NGAL originates not only from the injured kidney but also from leucocytes and liver.

Our results agree with $\mathrm{Kim} \mathrm{H}$ et al, 2013 who declared that Plasma NGAL was significantly higher in septic patients than in non-septic patients (337.5 vs. $129.0 \mathrm{mg} / \mathrm{ml}, \mathrm{p}<0.0001$ ). Plasma NGAL was also significantly higher in AKI patients than in nonAKI patients. However, there was no significant difference in plasma NGAL between septic patients with AKI and those without AKI. NGAL correlated well with procalcitonin in sepsis diagnosis and staging. These findings suggest the great value of NGAL in sepsis, although a prospective randomised trial would provide further validation.

Our results agree with that of Bagshaw et al (Bagshaw et al., 2010) who found that plasma and urinary NGAL levels to be substantially higher in patients with septic AKI compared to nonseptic AKI. This fact may limit the diagnostic utility of NGAL in septic patients. Bagshaw et al., (2007).Confirmed that Sepsis might hamper the predictive property of serum NGAL as a biomarker of AKI in the general intensive care unit (ICU) setting.

In contrast to our results, Wheeler et al., (2008) stated that serum NGAL is significantly increased within the first 24 hours and remains increased at day 3 following admission to the pediatric ICU in critically ill children who develop AKI compared to critically ill children who do not develop AKI. They reported a sensitivity $=86 \%$ and specificity $=39 \%$ and concluded that serum NGAL is a high sensitive but a non-specific predictor of AKI in critically ill children with septic shock.
Zappitelli et al., (2007) hypothesized that sepsis induced a greater "injury" to the kidney compared with other contributing factors, and higher plasma NGAL levels have been associated with greater severity of AKI.

In our study, ROC analysis showed that serum NGAL had a poor sensitivity with poor specificity for prediction of AKI, and their ability to predict AKI in patients with septic shock was poor with an AUC-ROC $=0.56$. In accordance to our result, Wheeler et al., (2008) and Martensson et al., (2010) found that the ability of plasma NGAL to predict AKI in patients with septic shock was poor with AUC-ROC $(0.68$ and 0.67 respectively).Meanwhile, ROC analysis of serum creatinine in our study showed a higher sensitivity and higher specificity for prediction of AKI, so serum creatinine has better ability to predict AKI in septic patients.

\section{Conclusion}

We concluded that serum NGAL is raised in critically ill septic children in pediatric ICU and is a marker of bacterial infection and systemic inflammation. However, in AKI associated with sepsis, serum NGAL is not a specific biomarker for the prediction of AKI and it loses its early predictive property. In such patients, serum creatinine is more sensitive and specific than serum NGAL.

\section{References}

1. Acute Kidney Injury Work Group. Kidney Disease Improving Global Outcomes (KDIGO) - Clinical Practice Guideline for Acute Kidney Injury. Kidney Inter. 2012;2:1-138.

2. Alkandari O, Eddington KA, Hyder A, Gauvin F, Ducruet T, Gottesman R, Phan V, Zappitelli M. Acute kidney injury is an independent risk factor for 
pediatric intensive care unit mortality, longer length of stay and prolonged mechanical ventilation in critically ill children: a two-center retrospective cohort study. Crit Care. 2011; 15: R146.

3. Bagshaw SM, Bennett M, Haase M, Haase-Fielitz A, Egi M, Morimatsu H, d'Amico G, Goldsmith D, Devarajan P, Bellomo R: Plasma and urine neutrophil gelatinase-associated lipocalin in septic versus non-septic acute kidney injury in critical illness. Intensive Care Med 2010;36:452-461.

4. Bagshaw, S.M., S. Uchino, R. Bellomo, H. Morimatsu, S. Morgera, et al. Septic acute kidney injury in critically ill patients: clinical characteristics and outcomes. Clin J Am Soc Nephrol.2007, 2: 431-439.

5. Consuelo Restrepo de Rovetto, Julián Andrés Mora, Sergio Alexandre Cardona, Andrés Felipe Marmolejo, Juan Fernando Paz, Iris de Castaño. Acute kidney injury applying pRifle scale in Children of Hospital Universitario del Valle in Cali, Colombia: clinical features, management and evolution. Colombia medica.2012; Vol 43, No 3.

6. Devarajan P. Biomarkers for the early detection of acute kidney injury. CurrOpinPediatr. 2011;23(2):194-200.

7. Goldstein, B., B. Giroir, A. Randolph. International pediatric sepsis consensus conference: definitions for sepsis and organ dysfunction in pediatrics. PediatrCrit Care Med.2005; 6(1): 2-8.

8. Hanley, J.A., B.A. McNeil. The meaning and use of the area under a receiver operating (ROC) curve. Radiology.1982; 143: 29-36.

9. Kawasaki Tatsuya. Update on pediatric sepsis: a review. Journal of Intensive Care 2017; 5:47.

10.Kim H, Hur M, Cruz DN, Moon HW, Yun YM: Plasma neutrophil gelatinaseassociated lipocalin as a biomarker for acute kidney injury in critically ill patients with suspected sepsis. ClinBiochem 2013;46:1414-1418.
11.Kjeldsen, L., J.B. Cowland, N. Borregaard. Human neutrophil gelatinase-associated lipocalin and homologous proteins in rat and mouse. BiochimBiophys Acta.2000; 1482(1-2): 272-283.

12.Krishnamurthy S. Incidence and etiology of acute kidney injury in Southern India: author's reply. Indian J Pediatr. 2013; 80: 797.

13.Martensson, J., B. Max, O. Anders, X. Shengyuan, et al. Neutrophil gelatinase-associated lipocalin in adult septic patients with and without acute kidney injury.Intensive Care Med.2010; 36(8): 1333-1340.

14.Mazzachi, B.C., M.J. Peake, V. Ehrhardt. Reference range and method comparison studies for enzymatic and Jaffe' creatinine assay in plasma and serum and early morning urine.Clin Lab.2000; 46: 53-55.

15.Mori, K., H.T. Lee, D. Rapoport, I.R. Drexler, et al. Endocytic delivery of lipocalinsiderophore-iron complex rescues the kidney from ischemiareperfusion injury. J Clin Invest. 2005; 115: 610-621.

16.Mori, K., K. Nakao. Neutrophil gelatinase-associated lipocalin as the real-time indicator of active kidney damage. Kidney Int.2007; 71(10): 96770.

17.Pollack, M.M., K.M. Patel, U.E. Ruttimann. PRISM III: An updated Pediatric Risk of Mortality score.Crit Care Med.1996; 24(5): 743-752.

18. Shalaby Mohamed, Norah Khathlan, Osama Safder, FatinaFadel, Youssef M.K. Farag, Ajay K. Singh, and Jameela A. Kari. Outcome of acute kidney injury in pediatric patients admitted to the intensive care unit. Clinical Nephrology2014, Vol. 82 No. 6 (379-386).

19.Singer Eugenia, LajosMarkó, Neal Paragas, Jonathan Barasch, DuskaDragun, Dominik N. Müller, KlemensBudde, and Kai M. SchmidtOtt. Neutrophil gelatinase-associated lipocalin: pathophysiology and clinical 
SOHAG MEDICAL JOURNAL Serum Neutrophil Gelatinase-associated Lipocalin Vol. 23 No.2Apr 2019

Mohamed A. Kassem.et al

applications. ActaPhysiol (Oxf). 2013 Apr; 207(4): 663-672.

20.Wagener, G., M. Jan, M. Kim, K. Mori, J.M. Barasch, et al. Association between increases in urinary neutrophil gelatinase-associated lipocalin and acute renal dysfunction after adult cardiac surgery. Anesthesiology 2006; 105: 485-491.

21. Waikar S, Betensky R, Bonventre J: Creatinine as the gold standard for kidney injury biomarker studies? Nephrol Dial Transplant 2009, 24:3265-3268.
22. Wheeler, D.S., P. Devarajan, Q. Ma, K. Harmon, et al. Serum neutrophil gelatinase-associated lipocalin (NGAL) as a marker of acute kidney injury in critically ill children with septic shock. Crit Care Med.2008; 36: 1297-1303.

23.Zappitelli, M., K.K. Washburn, A.A. Arikan, L. Loftis, et al. Urine neutrophil gelatinase-associated lipocalin is an early marker of acute kidney injury in critically ill children: a prospective cohort study. Crit Care.2007; 11: R84. 